\title{
The Method of Lines for Ternary Diffusion Problems
}

\author{
Henryk Leszczyński and Milena Matusik \\ Institute of Mathematics, University of Gdańsk, Wit Stwosz Street 57, 80-952 Gdańsk, Poland \\ Correspondence should be addressed to Henryk Leszczyński; hleszcz@mat.ug.edu.pl
}

Received 9 December 2013; Revised 26 February 2014; Accepted 3 March 2014; Published 28 April 2014

Academic Editor: Alvaro H. Salas

Copyright (C) 2014 H. Leszczyński and M. Matusik. This is an open access article distributed under the Creative Commons Attribution License, which permits unrestricted use, distribution, and reproduction in any medium, provided the original work is properly cited.

The method of lines (MOL) for diffusion equations with Neumann boundary conditions is considered. These equations are transformed by a discretization in space variables into systems of ordinary differential equations. The proposed ODEs satisfy the mass conservation law. The stability of solutions of these ODEs with respect to discrete $L^{2}$ norms and discrete $W^{1, \infty}$ norms is investigated. Numerical examples confirm the parabolic behaviour of this model and very regular dynamics.

\section{Introduction}

Diffusion is one of several transport phenomena that occur in nature. Many physical processes in metallurgy are controlled by diffusion, for example, oxidation and sintering. There are two ways to introduce the notion of diffusion: an atomistic approach and continuum approach. The central role in the diffusion theory of multicomponent systems plays the Kirkendall effect [1], i.e., the discovery that drift in solids can be generated by diffusion. The explanation of the effect and an original method of its mathematical analysis was proposed by Darken [2]. The Kirekndall effect describes what happens when two solids diffuse into each other at different rates. This subject is treated in many textbooks on solid-state and publications [3-7]. In [8,9] the authors deal with stability of Kirkendall planes. The peculiarities of the deformation in one-dimensional binary diffusion couple with a moving interface are described in [10].

Our ternary system arises from the Fick second law. If the overall molar density $c=c(t, x)$ and component molar ratio $N_{i}=N_{i}(t, x)$ are defined by

$$
c=\sum_{i=1}^{3} c_{i}
$$

( $c_{i}$ is the molar concentration of the $i$ th component in the mixture, $\left.\left[\mathrm{mole} / \mathrm{dm}^{3}\right]\right)$

$$
N_{i}=\frac{c_{i}}{c}
$$

where this quatity is a ratio of $i$ th component atoms to averall number of atoms. Then we have

$$
\frac{\partial N_{i}}{\partial t}=D_{i} \frac{\partial^{2} N_{i}}{\partial x^{2}}-\frac{\partial\left(N_{i} \nu^{D}\right)}{\partial x}, \quad i=1,2,3
$$

where $D_{i}$ denotes the intrinsic diffusivity of the $i$ th component and $v^{D}$ is the drift velocity. In the paper $N_{i}, i=1,2,3$ will be denoted by $u, v$, and $w$, respectively.

The method proposed by Darken [2], was extended by Danielewski et al. in [11, 12]. Their generalization of Darken's method describes the interdiffusion process in bounded mixtures with constant concentrations and with variable diffusivities of the elements.

We are interested in establishing an approximation method of solutions to the ternary system mentioned above by solutions of associated systems of ordinary differential equations. These systems of ordinary differential equations are obtained by using a discretization in spatial variables.

From the abundant literature concerning the numerical method of lines (MOL) for classical PDEs we mention the monographs [13-15]. MOL for time-dependent onedimensional systems of parabolic partial differential equations is proposed in [16]. Numerical examples for problems such as Burger's equation or nonlinear diffusion equation with nonlinear boundary conditions were presented in [16]. 
A posteriori error estimates for evolution problems (general nonlinear parabolic problem with a strongly monotonic elliptic operator, a linear nonstationary convectiondiffusion problem, and linear second-order hyperbolic problem) solved by the method of lines are derived in [17].

The aim of the paper is to construct a method of lines for diffusion equations in three-component system with Neumann boundary conditions. We prove stability of approximate solutions with respect to discrete $L^{2}$ norms and discrete $W^{1, \infty}$ norms.

Our research is concerned with the situation where the initial data and solutions are at least of class $C^{2}$. This regularity is assumed throughout the paper. It is observed that even very irregular data in a short time give highly smooth solutions (concentrations of the components). Moreover, these concentrations are mixed, which means that they are inside the interval $(0,1)$ for $t>0$. Therefore, we assume that the initial data are of class $C^{2}$. This observation also justifies our assumptions that the Lipschitz constants and second derivatives of solutions are bounded by some constant independent of the discretization parameter. Under these assumptions we prove that solutions of ODEs satisfy the maximum principle and remain inside the interval $(0,1)$. We also show convergence and stability of the approximate solutions. Our results confirm the parabolic nature of the equations in question. The existence and uniqueness of solutions of the interdiffusion problem in $W^{1,2}$ space was shown by the variational methods in [12]. In [18] authors constructed an example of an interdiffusion equation that does not fulfill a peculiar type of parabolicity, meant in the sense of a decreasing $L^{2}$ norm. In Section 4 we show the convergence of MOL in discrete $W^{1, \infty}$ norms, while in Section 3 we attempt to show convergence in discrete $L^{2}$ norms. It turns out that we have stability in $L^{2}$ norms for certain values of the diffusion coefficients $D_{1}, D_{2}, D_{3}$, which highlights the parabolic nature of the equation.

In Section 5 we present numerical examples where we consider Darken's trajectory besides solutions. Since the right-hand sides of ODEs are polynomial, we attempt in the appendix to estimate the radius of convergence of Taylor series solutions. It turns out that this radius is proportional to $h^{2}$. This means that Cauchy-Kovalevskaya theorem has limited (restricted) applicability to our system. Because R$\mathrm{K}$ methods are close to Taylor expansions, our observation points out that one should be careful with these methods.

\section{Formulation of the Problem}

We consider the system of diffusion equations:

$$
\begin{aligned}
\frac{\partial u}{\partial t} & =D_{1} \frac{\partial^{2} u}{\partial x^{2}}-\frac{\partial}{\partial x}\left(\begin{array}{ll}
u & v^{D}
\end{array}\right), \\
\frac{\partial v}{\partial t} & =D_{2} \frac{\partial^{2} v}{\partial x^{2}}-\frac{\partial}{\partial x}\left(\begin{array}{ll}
v & v^{D}
\end{array}\right), \\
\frac{\partial w}{\partial t} & =D_{3} \frac{\partial^{2} w}{\partial x^{2}}-\frac{\partial}{\partial x}\left(\begin{array}{ll}
w & v^{D}
\end{array}\right),
\end{aligned}
$$

on $[0, T] \times[-L, L] \subset \mathbb{R}^{2}$ with the initial boundary conditions

$$
\begin{gathered}
u(0, x)=u_{0}(x), \\
v(0, x)=v_{0}(x), \\
w(0, x)=w_{0}(x) \\
\text { for } x \in[-L, L] \\
\frac{\partial u}{\partial x}=0, \quad \frac{\partial v}{\partial x}=0, \quad \frac{\partial w}{\partial x}=0 \quad \text { for } x= \pm L,
\end{gathered}
$$

where the diffusion coefficients $D_{1}, D_{2}, D_{3}$ are different and positive, say $D_{1}>D_{2}>D_{3}>0, u_{0}, v_{0}, w_{0} \in C^{2}\left([-L, L], \mathbb{R}_{+}\right)$, and $u_{0}+v_{0}+w_{0}=1$ for $x \in[-L, L]$, and

$$
v^{D}=D_{1} \frac{\partial u}{\partial x}+D_{2} \frac{\partial v}{\partial x}+D_{3} \frac{\partial w}{\partial x},
$$

is the drift velocity. These data imply $u+v+w \equiv 1$.

We formulate the method of lines corresponding to (4) on a regular mesh with step $h$ such that $N h=L$. The difference operators $\delta^{(2)}, \delta^{+}, \delta^{-}$are defined in the following way:

$$
\begin{gathered}
\delta^{+} u^{(i)}=\frac{u^{(i+1)}-u^{(i)}}{h}, \quad \delta^{-} u^{(i)}=\frac{u^{(i)}-u^{(i-1)}}{h}, \\
\delta^{(2)} u^{(i)}=\frac{u^{(i+1)}-2 u^{(i)}+u^{(i-1)}}{h^{2}}=\delta^{+} \delta^{-} u^{(i)}
\end{gathered}
$$

Denote by $\mathbf{v}^{(j)}$ the discrete drift velocity $\mathbf{v}^{(j)}=D_{1} \delta^{+} u^{(j)}+$ $D_{2} \delta^{+} v^{(j)}+D_{3} \delta^{+} w^{(j)}$. We will consider the following ODE system:

$$
\begin{aligned}
\frac{d}{d t} u^{(j)}= & D_{1} \delta^{(2)} u^{(j)}-u^{(j)} \delta^{-} \mathbf{v}^{(j)} \\
& -\frac{1}{2} \delta^{+} u^{(j)} \cdot \mathbf{v}^{(j)}-\frac{1}{2} \delta^{-} u^{(j)} \cdot \mathbf{v}^{(j-1)} \\
\frac{d}{d t} v^{(j)}= & D_{2} \delta^{(2)} v^{(j)}-v^{(j)} \delta^{-} \mathbf{v}^{(j)} \\
& -\frac{1}{2} \delta^{+} v^{(j)} \cdot \mathbf{v}^{(j)}-\frac{1}{2} \delta^{-} v^{(j)} \cdot \mathbf{v}^{(j-1)} \\
\frac{d}{d t} w^{(j)}= & D_{3} \delta^{(2)} w^{(j)}-w^{(j)} \delta^{-} \mathbf{v}^{(j)} \\
& -\frac{1}{2} \delta^{+} w^{(j)} \cdot \mathbf{v}^{(j)}-\frac{1}{2} \delta^{-} w^{(j)} \cdot \mathbf{v}^{(j-1)}
\end{aligned}
$$

for $j=-N, \ldots, N$ with the initial conditions

$$
\begin{array}{r}
u^{(j)}(0)=u_{0}(h j) \geq 0, \\
v^{(j)}(0)=v_{0}(h j) \geq 0, \\
w^{(j)}(0)=w_{0}(h j) \geq 0, \\
j=-N, \ldots, N .
\end{array}
$$


The discrete Neumann boundary conditions

$$
\begin{aligned}
u^{(N+1)}=u^{(N-1)}, & v^{(N+1)}=v^{(N-1)}, \\
w^{(N+1)}=w^{(N-1)}, & u^{(-N-1)}=u^{(-N+1),} \\
v^{(-N-1)}=v^{(-N+1)}, & w^{(-N-1)}=w^{(-N+1)}
\end{aligned}
$$

can be regarded as a convenient definition of auxiliary quantities: $u^{(N+1)}, v^{(N+1)}, w^{(N+1)}, u^{(-N-1)}, v^{(-N-1)}, w^{(-N-1)}$.

Remark 1. Local existence and uniqueness of solutions of (8) with the above conditions follows from the general theory of ODE systems.

Suppose that we have two constants $M_{0}, M$, independent of $h$, satisfying the inequality $M \geq M_{0}>0$. This assumption is valid throughout the paper. $M_{0}$ will be the Lipschitz constant for the initial functions $u_{0}, v_{0}, w_{0} . M$ will play the same role for $u, v, w$

$$
\begin{aligned}
\mathfrak{X}_{0}=\{ & \left(u_{0}, v_{0}, w_{0}\right) \in \mathbb{R}^{3(2 N+1)}: u_{0}^{(j)}, v_{0}^{(j)}, w_{0}^{(j)} \geq 0, \\
& u_{0}^{(j)}+v_{0}^{(j)}+w_{0}^{(j)}=1 \text { for } j=-N, \ldots, N \\
& \left|u_{0}^{(i)}-u_{0}^{(j)}\right| \leq M_{0}|i-j| h,\left|v_{0}^{(i)}-v_{0}^{(j)}\right| \leq M_{0}|i-j| h \\
& \left.\left|w_{0}^{(i)}-w_{0}^{(j)}\right| \leq M_{0}|i-j| h\right\}, \\
\mathfrak{X}=\{ & (u, v, w):[0, T] \longrightarrow \mathbb{R}^{3(2 N+1)}: u^{(j)}, v^{(j)}, w^{(j)} \geq 0, \\
& u^{(j)}+v^{(j)}+w^{(j)}=1 \text { for } j=-N, \ldots, N \\
& \left|u^{(i)}-u^{(j)}\right| \leq M|i-j| h,\left|v^{(i)}-v^{(j)}\right| \leq M|i-j| h \\
& \left.\left|w^{(i)}-w^{(j)}\right| \leq M|i-j| h\right\} .
\end{aligned}
$$

We will refer to these Lipschitz-type inequalities in the definitions of $\mathfrak{X}_{0}$ and $\mathfrak{X}$ as $M_{0}$-Lipschitz and $M$-Lipschitz, respectively. It is assumed that approximate solutions are Lipschitz continuous with respect to spatial variable. The Lipschitz continuity of approximate solutions can be proved rigorously; however, we omit the proof for brevity. To justify our proceeding, consider solutions of ternary systems. They are bounded together with their first and second derivatives.

If the initial data belong to $\mathfrak{X}_{0}$ the equations for $w$ can be eliminated from (8). The following lemma allows us to express $(8)$ as the system of $2(2 N+1)$ ordinary differential equations.

Lemma 2. Suppose that $(u, v, w)$ is the solution of (8) with the initial condition $\left(u_{0}, v_{0}, w_{0}\right) \in \mathfrak{X}_{0}$. Assume that $u, v, w$ are $M$ Lipschitz. Then $(u, v, w) \in \mathfrak{X}$.
Proof. First, we prove that $u^{(j)}+v^{(j)}+w^{(j)}=1$ for $j=$ $-N, \ldots, N$. Let $g=u+v+w$. We have

$$
\begin{aligned}
\frac{d}{d t}\left(g^{(j)}\right)= & {\left[1-g^{(j)}\right] \delta^{-} \mathbf{v}^{(j)} } \\
& -\frac{1}{2} \delta^{+}\left(g^{(j)}\right) \cdot \mathbf{v}^{(j)}-\frac{1}{2} \delta^{-}\left(g^{(j)}\right) \cdot \mathbf{v}^{(j-1)},
\end{aligned}
$$

for $j=-N, \ldots, N$ with the initial condition $g^{(j)}(0)=1$ and the discrete Neumann boundary conditions. It follows from the general ODE theory that there exists exactly one solution $g^{(j)} \equiv 1$.

We next show that $u^{(j)}, v^{(j)}, w^{(j)} \geq 0$ on $[0, T]$ for $j=$ $-N, \ldots, N$. Suppose first that $u^{(j)}(0), v^{(j)}(0), w^{(j)}(0)>0$. We claim that $u^{(j)}, v^{(j)}, w^{(j)}>0$ on $[0, T]$ for $j=-N, \ldots, N$. To obtain a contradiction, we suppose that there is $\widetilde{t}>0$ such that $u^{(j)}(t), v^{(j)}(t), w^{(j)}(t)>0$ for $t<\tilde{t}$ and $j=-N, \ldots, N$ and there is $i \in\{-N, \ldots, N\}$ such that $u^{(i)}(\widetilde{t})=0$. We have $u^{(j)}(\widetilde{t}), v^{(j)}(\widetilde{t}), w^{(j)}(\widetilde{t}) \geq 0$ for $j=-N, \ldots, N$. Then

$$
\begin{gathered}
\frac{d}{d t} u^{(i)}(\widetilde{t}) \leq 0, \quad \delta^{+} u^{(i)}(\widetilde{t}) \geq 0, \\
\delta^{-} u^{(i)}(\widetilde{t}) \leq 0 .
\end{gathered}
$$

We exploit (8) at the point $\widetilde{t}$ to get the following inequality:

$$
\begin{aligned}
0 \geq \frac{d}{d t} u^{(i)}(\widetilde{t})= & D_{1} \delta^{(2)} u^{(i)}(\widetilde{t})-\frac{1}{2} \delta^{+} u^{(i)}(\widetilde{t}) \mathbf{v}^{(i)} \\
& -\frac{1}{2} \delta^{-} u^{(i)}(\widetilde{t}) \mathbf{v}^{(i-1)} \\
= & \delta^{+} u^{(i)}(\widetilde{t})\left[\frac{D_{1}}{h}-\mathbf{v}^{(i)}\right] \\
& -\delta^{-} u^{(i)}(\widetilde{t})\left[\frac{D_{1}}{h}+\mathbf{v}^{(i-1)}\right] .
\end{aligned}
$$

The expressions in the brackets are positive for small $h$. Hence

$$
\delta^{+} u^{(i)}(\tilde{t})=0, \quad \delta^{-} u^{(i)}(\tilde{t})=0 .
$$

We see at once that $u^{(i)}(\widetilde{t})=0$ for $i=-N, \ldots, N$. It follows from the general ODE theory [19, page 108] that $u(t)=0$ for $t \in[0, T]$, contrary to strict initial inequalities. This argument also settles the case of $v^{(i)}(\widetilde{t})=0$ or $w^{(i)}(\widetilde{t})=0$. We now turn to the case $u(0, \cdot), v(0, \cdot), w(0, \cdot) \geq 0$. It follows from the theorem on the continuous dependence on initial data [19, page 145] that $u^{(j)}, v^{(j)}, w^{(j)} \geq 0$ for $j=-N, \ldots, N$.

Denote by $h \sum_{j}^{\prime}$ the trapezoidal sum. More precisely,

$$
h \sum_{j}^{\prime} u^{(j)}=h \sum_{j=-N}^{N-1} \frac{u^{(j)}+u^{(j+1)}}{2} .
$$

This quantity corresponds to the total mass of $u$. The MOL system is conservative. It preserves the mass of $u, v$. 
Lemma 3. Suppose that $u, v, w$ are solutions of (8) such that $\left(u_{0}, v_{0}, w_{0}\right) \in \mathfrak{X}_{0}$. Then

$$
h \sum_{j}^{\prime} u^{(j)}=\text { const, } \quad h \sum_{j}^{\prime} v^{(j)}=\text { const. }
$$

Proof. Note that $\delta^{+} u^{(j)}=\delta^{-} u^{(j+1)}$ for $j=-N, \ldots N$. Hence

$$
\begin{gathered}
h \sum_{j}^{\prime} D_{1} \delta^{(2)} u^{(j)}=h \sum_{j}^{\prime}\left[D_{1} \delta^{+} u^{(j)}-D_{1} \delta^{+} u^{(j-1)}\right]=0 \\
h \sum_{j}^{\prime}\left(-u^{(j)} \delta^{-} \mathbf{v}^{(j)}-\frac{\delta^{+} u^{(j)}}{2} \cdot \mathbf{v}^{(j)}-\frac{\delta^{-} u^{(j)}}{2} \cdot \mathbf{v}^{(j-1)}\right) \\
=\frac{h D_{1}^{\prime}}{2} \sum_{j}^{\prime}\left(-u^{(j)} \delta^{+} u^{(j)}+u^{(j)} \delta^{+} u^{(j-1)}\right. \\
\left.-u^{(j+1)} \delta^{+} u^{(j)}+u^{(j-1)} \delta^{+} u^{(j-1)}\right) \\
+\frac{h D_{2}^{\prime}}{2} \sum_{j}^{\prime}\left(-u^{(j)} \delta^{+} v^{(j)}+u^{(j)} \delta^{+} v^{(j-1)}\right. \\
\left.-u^{(j+1)} \delta^{+} v^{(j)}+u^{(j-1)} \delta^{+} v^{(j-1)}\right)=0
\end{gathered}
$$

where $D_{1}^{\prime}=D_{1}-D_{3}$ and $D_{2}^{\prime}=D_{2}-D_{3}$. It follows from these equations that

$$
\frac{d}{d t}\left(h \sum_{j}^{\prime} u^{(j)}\right)=h \sum_{j}^{\prime} \frac{d}{d t} u^{(j)}=0
$$

\section{Stability with respect to Discrete $L^{2}$ Norms}

The stability of the method of lines with respect to discrete $L^{2}$ norm is established by the following theorem.

\section{Theorem 4. Suppose that}

(1) $(u, v, w) \in \mathfrak{X}$ is the unique solution of system (8) with the initial condition $\left(u_{0}, v_{0}, w_{0}\right) \in \mathfrak{X}_{0}$,

(2) $(\bar{u}, \bar{v}, \bar{w}) \in \mathfrak{X}$ is an approximate solution of (8) with the initial condition $\left(\bar{u}_{0}, \bar{v}_{0}, \bar{w}_{0}\right) \in \mathfrak{X}_{0}$ and with perturbations of the right-hand sides denoted by $\xi_{1}^{(j)}$, $\xi_{2}^{(j)}, \xi_{3}^{(j)}$ for $j=-N, \ldots, N$,

(3) consider

$$
\begin{aligned}
& -D_{2}^{2}+14 D_{1} D_{2}-D_{1}^{2}>0 \\
& -D_{3}^{2}+14 D_{1} D_{3}-D_{1}^{2}>0 \\
& -D_{3}^{2}+14 D_{2} D_{3}-D_{2}^{2}>0
\end{aligned}
$$

Then we have

$$
A_{t} \leq C_{1}\left[A_{0}+\|\xi\|\right] \exp \left(K_{1} t\right)
$$

where $K_{1}, C_{1}>0$ are constants independent of $h$ and

$$
\|\xi\|=\max _{0 \leq t \leq T} h \sum_{j}^{\prime}\left[\left(\xi_{1}^{(j)}(t)\right)^{2}+\left(\xi_{2}^{(j)}(t)\right)^{2}+\left(\xi_{3}^{(j)}(t)\right)^{2}\right]
$$

$$
\begin{aligned}
A_{t}=h \sum_{j}^{\prime}[ & \left(\bar{u}^{(j)}(t)-u^{(j)}(t)\right)^{2} \\
& +\left(\bar{v}^{(j)}(t)-v^{(j)}(t)\right)^{2} \\
& \left.+\left(\bar{w}^{(j)}(t)-w^{(j)}(t)\right)^{2}\right] .
\end{aligned}
$$

Proof. Denote by $\Delta u, \Delta v, \Delta w$ the differences between the approximate and exact solutions of (8).

The proof is based on the following observation:

$$
\begin{aligned}
\frac{d}{d t} A_{t}=2 h \sum_{j}^{\prime}\left[\Delta u^{(j)} \frac{d}{d t}\left(\bar{u}^{(j)}-u^{(j)}\right)\right. & \\
& +\Delta v^{(j)} \frac{d}{d t}\left(\bar{v}^{(j)}-v^{(j)}\right) \\
& \left.+\Delta w^{(j)} \frac{d}{d t}\left(\bar{w}^{(j)}-w^{(j)}\right)\right] \\
= & h \sum_{j}^{\prime} 2 \Delta u^{(j)}\left\{D_{1} \delta^{(2)} \Delta u^{(j)}-\bar{u}^{(j)} \delta^{-} \overline{\mathbf{v}}^{(j)}+u^{(j)} \delta^{-} \mathbf{v}^{(j)}\right. \\
& -\frac{1}{2} \delta^{+} \bar{u}^{(j)} \cdot \overline{\mathbf{v}}^{(j)}+\frac{1}{2} \delta^{+} u^{(j)} \cdot \mathbf{v}^{(j)} \\
& \left.-\frac{1}{2} \delta^{-} \bar{u}^{(j)} \cdot \overline{\mathbf{v}}^{(j-1)}+\frac{1}{2} \delta^{-} u^{(j)} \cdot \mathbf{v}^{(j-1)}\right\} \\
& +h \sum_{j}^{\prime} 2 \Delta v^{(j)}\left\{\frac{d}{d t}\left(\bar{v}^{(j)}-v^{(j)}\right)\right\} \\
& +h \sum_{j}^{\prime} 2 \Delta w^{(j)}\left\{\frac{d}{d t}\left(\bar{w}^{(j)}-w^{(j)}\right)\right\} \\
& \left.+h u^{(j)} \xi_{1}^{(j)}+2 \Delta v^{(j)} \xi_{2}^{(j)}+2 \Delta w^{(j)} \xi_{3}^{(j)}\right\} \cdot
\end{aligned}
$$

The terms $(d / d t)\left(\bar{v}^{(j)}-v^{(j)}\right)$ and $(d / d t)\left(\bar{w}^{(j)}-w^{(j)}\right)$ are calculated similarly as $(d / d t)\left(\bar{u}^{(j)}-u^{(j)}\right)$. Set

$$
\begin{gathered}
Z_{1}=\delta^{+} \Delta u^{(j)}(t), \quad Z_{2}=\delta^{+} \Delta v^{(j)}(t), \\
Z_{3}=\delta^{+} \Delta w^{(j)}(t) .
\end{gathered}
$$


We apply the summation by parts and obtain

$$
\begin{aligned}
\frac{d}{d t} A_{t}=h \sum_{j=-N}^{N-1}\{ & 2 D_{1}\left(Z_{1}\right)^{2}-2 D_{2}\left(Z_{2}\right)^{2}-2 D_{3}\left(Z_{3}\right)^{2} \\
& +Z_{1}\left(\Delta u^{(j+1)}+\Delta u^{(j)}\right) \cdot \overline{\mathbf{v}}^{(j)} \\
& +Z_{2}\left(\Delta v^{(j+1)}+\Delta v^{(j)}\right) \cdot \overline{\mathbf{v}}^{(j)} \\
& +Z_{3}\left(\Delta w^{(j+1)}+\Delta w^{(j)}\right) \cdot \overline{\mathbf{v}}^{(j)} \\
& +D_{1}\left(Z_{1}\right)^{2}\left(u^{(j+1)}+u^{(j)}\right) \\
& +D_{2} Z_{1} Z_{2}\left(u^{(j+1)}+u^{(j)}\right) \\
& +D_{3} Z_{1} Z_{3}\left(u^{(j+1)}+u^{(j)}\right) \\
& +D_{1} Z_{1} Z_{2}\left(v^{(j+1)}+v^{(j)}\right) \\
& +D_{2}\left(Z_{2}\right)^{2}\left(v^{(j+1)}+v^{(j)}\right) \\
& +D_{3} Z_{2} Z_{3}\left(v^{(j+1)}+v^{(j)}\right) \\
& +D_{1} Z_{1} Z_{3}\left(w^{(j+1)}+w^{(j)}\right) \\
& +D_{2} Z_{2} Z_{3}\left(w^{(j+1)}+w^{(j)}\right) \\
& \left.+D_{3}\left(Z_{3}\right)^{2}\left(w^{(j+1)}+w^{(j)}\right)\right\} \\
& \left.2 \Delta^{(j)} \xi_{1}^{(j)}+2 \Delta v^{(j)} \xi_{2}^{(j)}+2 \Delta w^{(j)} \xi_{3}^{(j)}\right\} \\
& \\
+h \sum_{j} & \\
& \\
&
\end{aligned}
$$

From Lemma 3 we have $Z_{3}=-Z_{1}-Z_{2}$. Set

$$
\begin{gathered}
2 A^{(i)}=\left(u^{(i+1)}+u^{(i)}\right), \quad 2 B^{(i)}=\left(v^{(i+1)}+v^{(i)}\right), \\
2 C^{(i)}=\left(w^{(i+1)}+w^{(i)}\right) .
\end{gathered}
$$

Hence the sign of $(d / d t) A_{t}$ depends on the quadratic form $T=\left[T_{i j}\right]_{i, j=1,2}$ :

$$
\begin{aligned}
& T_{11}=-2\left(D_{1}+D_{3}\right)+2\left(D_{1}-D_{3}\right)\left(A^{(i)}-C^{(i)}\right), \\
& T_{12}=T_{21}=-2 D_{3}+D_{2}^{\prime}\left(A^{(i)}-C^{(i)}\right)+D_{1}^{\prime}\left(B^{(i)}-C^{(i)}\right), \\
& T_{22}=-2\left(D_{2}+D_{3}\right)+2 D_{2}^{\prime}\left(B^{(i)}-C^{(i)}\right),
\end{aligned}
$$

which is negative definite for $D_{1}, D_{2}, D_{3}$ satisfying condition (20) of Theorem 4 . We have

$$
\begin{aligned}
& \frac{d}{d t} A_{t} \leq-K h \sum_{j=-N}^{N-1}\left(Z_{1}^{2}+Z_{2}^{2}+Z_{3}^{2}\right) \\
&+ h \sum_{j}^{\prime}\left(2 \Delta u^{(j)} \xi_{1}^{(j)}+2 \Delta v^{(j)} \xi_{2}^{(j)}+2 \Delta w^{(j)} \xi_{3}^{(j)}\right) \\
&+h \sum_{j=-N}^{N-1}\left\{Z_{1}\left(\Delta u^{(j+1)}+\Delta u^{(j)}\right)\right. \\
& \times\left(D_{1} \delta^{+} \bar{u}^{(j)}+D_{2} \delta^{+} \bar{v}^{(j)}+D_{3} \delta^{+} \bar{w}^{(j)}\right) \\
&+Z_{2}\left(\Delta v^{(j+1)}+\Delta v^{(j)}\right) \\
& \times\left(D_{1} \delta^{+} \bar{u}^{(j)}+D_{2} \delta^{+} \bar{v}^{(j)}+D_{3} \delta^{+} \bar{w}^{(j)}\right) \\
&+Z_{3}\left(\Delta w^{(j+1)}+\Delta w^{(j)}\right) \\
& \\
&\left.\quad \times\left(D_{1} \delta^{+} \bar{u}^{(j)}+D_{2} \delta^{+} \bar{v}^{(j)}+D_{3} \delta^{+} \bar{w}^{(j)}\right)\right\} \\
&+
\end{aligned}
$$

It follows from the inequality

$$
|a b| \leq \frac{1}{2}\left(K a^{2}+\frac{b^{2}}{K}\right) \quad \text { for } K>0
$$

that

$$
\begin{aligned}
\left|I_{2}\right| \leq & \frac{h}{K} \sum_{j}^{\prime}\left(\Delta u^{(j)}\right)^{2}+\left(\Delta v^{(j)}\right)^{2}+\left(\Delta w^{(j)}\right)^{2}+K\|\xi\| \\
\left|I_{3}\right| \leq & h M_{1}\left(D_{1}+D_{2}+D_{3}\right) \\
& \times \sum_{j=-N}^{N-1}\left(Z_{1} \Delta u^{(j+1)}+Z_{2} \Delta v^{(j+1)}+Z_{3} \Delta w^{(j+1)}\right) \\
& +h M_{1}\left(D_{1}+D_{2}+D_{3}\right) \\
& \times \sum_{j=-N}^{N-1}\left(Z_{1} \Delta u^{(j)}+Z_{2} \Delta v^{(j)}+Z_{3} \Delta w^{(j)}\right) \\
\leq & K h \sum_{j=-N}^{N-1}\left(Z_{1}^{2}+Z_{2}^{2}+Z_{3}^{2}\right) \\
& +\frac{h M_{1}^{2}\left(D_{1}+D_{2}+D_{3}\right)^{2}}{K} \\
& \times \sum_{j}^{\prime}\left(\left(\Delta u^{(j)}\right)^{2}+\left(\Delta v^{(j)}\right)^{2}+\left(\Delta w^{(j)}\right)^{2}\right) .
\end{aligned}
$$


Hence

$$
\begin{aligned}
\frac{d}{d t} A_{t} \leq & -K h \sum_{j=-N}^{N-1}\left(Z_{1}^{2}+Z_{2}^{2}+Z_{3}^{2}\right) \\
& +K h \sum_{j=-N}^{N-1}\left(Z_{1}^{2}+Z_{2}^{2}+Z_{3}^{2}\right) \\
& +\frac{\left(1+M_{1}^{2}\left(D_{1}+D_{2}+D_{3}\right)^{2}\right)}{K} A_{t}+K\|\xi\| .
\end{aligned}
$$

We conclude from this differential inequality that

$$
A_{t} \leq C_{1}\left(A_{0}+\|\xi\|\right) \exp \left(\frac{\left(1+M_{1}^{2}\left(D_{1}+D_{2}+D_{3}\right)^{2}\right)}{K} t\right)
$$

where

$$
C_{1}=\frac{K^{2}}{\left(1+M_{1}^{2}\left(D_{1}+D_{2}+D_{3}\right)^{2}\right)}
$$

This finishes the proof.

\section{Stability with respect to \\ Discrete $W^{1, \infty}$ Norms}

We define the norm $\|\cdot\|_{1, \infty}$ in $\mathbb{R}^{2 N+1}$ as follows:

$$
\|u\|_{1, \infty}=\|u\|_{\infty}+\left\|\delta^{+} u\right\|_{\infty}
$$

where $\|u\|_{\infty}=\max _{-N \leq j \leq N}\left|u^{(j)}\right|$ (with the discrete Neumann convention).

\section{Theorem 5. Suppose that}

(1) $(u, v, w) \in \mathfrak{X}$ is the exact solution of (8) with the initial condition $\left(u_{0}, v_{0}, w_{0}\right) \in \mathfrak{X}_{0}$,

(2) $(\bar{u}, \bar{v}, \bar{w}) \in \mathfrak{X}$ is an approximate solution of (8) with the initial condition $\left(\bar{u}_{0}, \bar{v}_{0}, \bar{w}_{0}\right) \in \mathfrak{X}_{0}$ and with perturbations of the right-hand sides denoted by $\xi_{1}^{(j)}$, $\xi_{2}^{(j)}, \xi_{3}^{(j)}$ for $j=-N, \ldots, N$.

Then we have

$$
\|\Delta u(t)\|_{1, \infty}+\|\Delta v(t)\|_{1, \infty} \leq \widetilde{K}\|\xi\|_{\infty} t^{1 / 2}
$$

where $\widetilde{K}$ is some constant independent of $h$.
Proof. It is easily seen that $\Delta u$ satisfies differential equation

$$
\begin{aligned}
\frac{d}{d t} \Delta u^{(i)}=[ & D_{1} \delta^{(2)} \Delta u^{(i)}-u^{(i)}\left(D_{1}^{\prime} \delta^{(2)} \Delta u^{(i)}+D_{2}^{\prime} \delta^{(2)} \Delta v^{(i)}\right) \\
& -\Delta u^{(i)}\left(D_{1}^{\prime} \delta^{(2)} \bar{u}^{(i)}+D_{2}^{\prime} \delta^{(2)} \bar{v}^{(i)}\right) \\
& -\frac{1}{2} \delta^{+} u^{(i)}\left(D_{1}^{\prime} \delta^{+} \Delta u^{(i)}+D_{2}^{\prime} \delta^{+} \Delta v^{(i)}\right) \\
& -\frac{1}{2} \delta^{+} \Delta u^{(i)}\left(D_{1}^{\prime} \delta^{+} \bar{u}^{(i)}+D_{2}^{\prime} \delta^{+} \bar{v}^{(i)}\right) \\
& -\frac{1}{2} \delta^{-} u^{(i)}\left(D_{1}^{\prime} \delta^{-} \Delta u^{(i)}+D_{2}^{\prime} \delta^{-} \Delta v^{(i)}\right) \\
& \left.-\frac{1}{2} \delta^{-} \Delta u^{(i)}\left(D_{1}^{\prime} \delta^{-} \bar{u}^{(i)}+D_{2}^{\prime} \delta^{-} \bar{v}^{(i)}\right)\right] \\
+ & \xi_{1}^{(i)}(t) .
\end{aligned}
$$

$\Delta v$ satisfies a similar differential equation.

Suppose that we have Green functions $G^{1, i, j}, G^{2, i, j}$ corresponding to the differential-difference operator:

$$
\left[\begin{array}{cc}
\frac{d}{d t}-D_{1} \delta^{(2)}+D_{1}^{\prime} u^{(i)} \delta^{(2)} & D_{2}^{\prime} u^{(i)} \delta^{(2)} \\
D_{1}^{\prime} v^{(i)} \delta^{(2)} & \frac{d}{d t}-D_{2} \delta^{(2)}+D_{2}^{\prime} v^{(i)} \delta^{(2)}
\end{array}\right]
$$

such that

$$
\begin{gathered}
h \sum_{j} G^{k, i, j}(t, s) \leq C, \\
h \sum_{j} \delta^{+} G^{k, i, j}(t, s) \leq \frac{C}{\sqrt{t-s}},
\end{gathered}
$$

where $C>0$ and $k=1,2$ (see [20]). Using the above Green functions we express the differences $\Delta u^{(i)}$ and $\Delta v^{(i)}$ as follows:

$$
\left[\begin{array}{l}
\Delta u^{(i)}(t) \\
\Delta v^{(i)}(t)
\end{array}\right]=\int_{0}^{t} h \sum_{j}\left[\begin{array}{l}
G^{1, i, j}(t, s) P^{1, j}(s) \\
G^{2, i, j}(t, s) P^{2, j}(s)
\end{array}\right] d s,
$$

where $P^{1, j}(t), P^{2, j}(t)$ depend on $\Delta u^{(j)}, \Delta v^{(j)}, \delta^{+} \Delta u^{(j)}$, $\delta^{+} \Delta u^{(j-1)}, \delta^{+} \Delta v^{(j)}, \delta^{+} \Delta v^{(j-1)}$ and satisfy the estimates

$$
\begin{aligned}
\left\|P^{i}(t)\right\|_{\infty} \leq & C_{0}\left(\|\Delta u(t)\|_{\infty}+\left\|\delta^{+} \Delta u(t)\right\|_{\infty}\right) \\
& +C_{0}\left(\|\Delta v(t)\|_{\infty}+\left\|\delta^{+} \Delta v(t)\right\|_{\infty}\right) \\
& +\left\|\xi_{i}(t)\right\|_{\infty}, \quad i=1,2,
\end{aligned}
$$




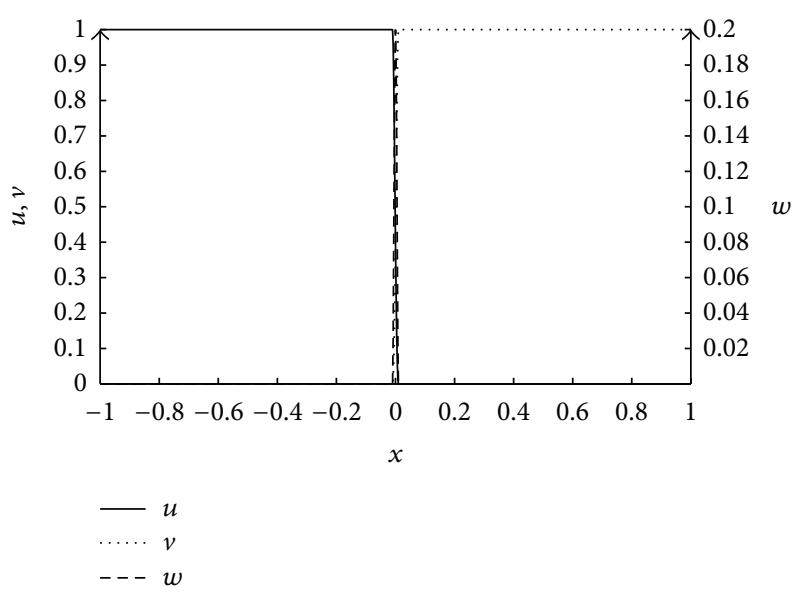

(a)

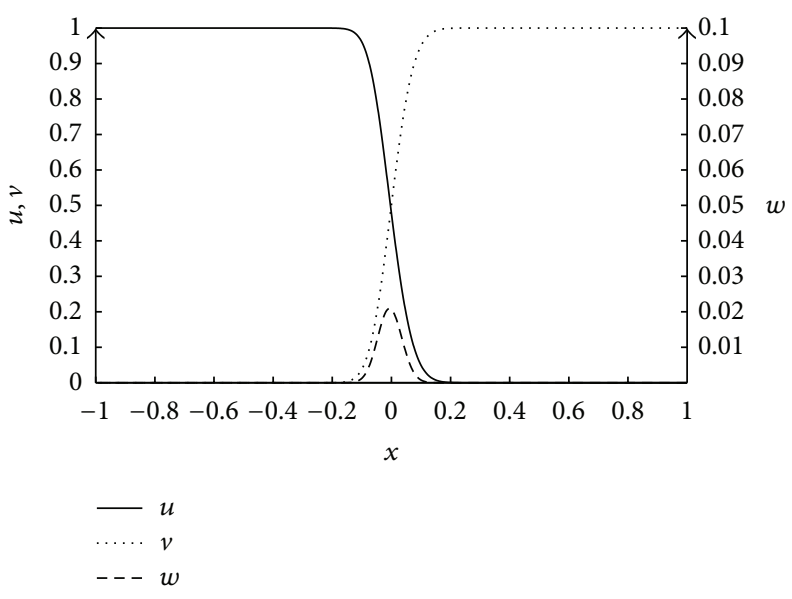

(b)

Figure 1: Initial values (a) and experimental values of $u, v$, and $w$ for $t=0.01, h=.002$ (b).

where $C_{0}$ depends on $M_{1}, D_{1}^{\prime}, D_{2}^{\prime}$. Hence

$$
\begin{aligned}
\|\Delta u(t)\|_{\infty} \leq & \int_{0}^{t} C\left\|P^{1}(s)\right\|_{\infty} d s \\
\leq & \int_{0}^{t} \frac{C}{\sqrt{t-s}}\left\|P^{1}(s)\right\|_{\infty} d s \\
\leq & \int_{0}^{t} \frac{C C_{0}}{\sqrt{t-s}} \\
& \times\left(\|\Delta u(s)\|_{1, \infty}+\|\Delta v(s)\|_{1, \infty}\right)+\|\xi\|_{\infty} d s, \\
\left\|\delta^{+} \Delta u(t)\right\|_{\infty} \leq & \int_{0}^{t} \frac{C}{\sqrt{t-s}}\left\|P^{1}(s)\right\|_{\infty} d s \\
\leq & \int_{0}^{t} \frac{C C_{0}}{\sqrt{t-s}} \\
& \quad \times\left(\|\Delta u(s)\|_{1, \infty}+\|\Delta v(s)\|_{1, \infty}\right)+\|\xi\|_{\infty} d s,
\end{aligned}
$$

where

$$
\|\xi\|_{\infty}=\max _{0 \leq t \leq T}\left\{\left\|\xi_{1}(t)\right\|_{\infty}+\left\|\xi_{2}(t)\right\|_{\infty}\right\}
$$

The same inequalities are satisfied by $\Delta v(t), \delta^{+} \Delta v(t)$. We get

$$
\begin{gathered}
\|\Delta u(t)\|_{\infty}+\left\|\delta^{+} \Delta u(t)\right\|_{\infty}+\|\Delta v(t)\|_{\infty}+\left\|\delta^{+} \Delta v(t)\right\|_{\infty} \\
\leq \int_{0}^{t} \frac{4 C C_{0}}{\sqrt{t-s}}\left(\|\Delta u(s)\|_{1, \infty}+\|\Delta v(s)\|_{1, \infty}+\|\xi\|_{\infty}\right) .
\end{gathered}
$$

It follows from Lemma A.1 that

$$
\|\Delta u(t)\|_{1, \infty}+\|\Delta v(t)\|_{1, \infty} \leq \widetilde{K}\|\xi\|_{\infty} t^{1 / 2}
$$

where $\widetilde{K}$ depends on $C, C_{0}$.

\section{Numerical Simulations}

Numerical Example 1. Set $L=1, D_{1}=0.2, D_{2}=0.15$, $D_{3}=0.08$ and the initial distributions $u, v$ are almost step functions, regularized near zero, whereas $w$ starts from a droplet:

$$
\begin{gathered}
u(0, x)=\Theta(100 x)-\Phi(100 x)+\mathbf{1}_{(.01,1]}(x), \\
\Theta(x)=\left(-\frac{1}{4} x^{3}+\frac{3}{4} x+\frac{9}{20}\right) \mathbf{1}_{[-1,1]}(x), \\
v(0, x)=\Theta(-100 x)-\Phi(100 x)+\mathbf{1}_{[-1,01)}(x), \\
w(0, x)=2 \cdot \Phi(100 x), \\
\Phi(x)=\left(\frac{1}{10} x^{4}-\frac{1}{5} x^{2}+\frac{1}{10}\right) \mathbf{1}_{[-1,1]}(x),
\end{gathered}
$$

for $x \in[-1,1]$ where 1 stands for a characteristic function. We use a second-order Runge-Kutta method with time step $h_{t}=2 \times 10^{-6}$. Here $D_{1}, D_{2}, D_{3}$ satisfy condition (20) of Theorem 4 . Note that $\left(u_{0}, v_{0}, w_{0}\right) \in \mathfrak{X}_{0}$ with $M=$ $M_{0}=30$. In Figure 1(a) we give experimental values of the solution $(u, v, w)$ of (8) for $t=0.01$ and $h=.002$ with the initial condition (45) (a). Since $h=.002$ we divide $[-1,1]$ into 1000 intervals. We put the values of $u, v, w$ at $t=0$. Note that $u_{0}$ is increasing on the interval $[-1,1]$. Furthermore $v_{0}$ is decreasing on the interval $[-1,1]$. The even function $w_{0}$ ("marker") is increasing on the interval $[-1,0]$ and decreasing on the interval $[0,1]$. We consider the differential equations describing the marker position $(d z / d t)(t)=$ $v^{D}(t, z(t))$, where $v^{D}$ is the Darken velocity. We use a secondorder Runge-Kutta method. The approximate position of the 


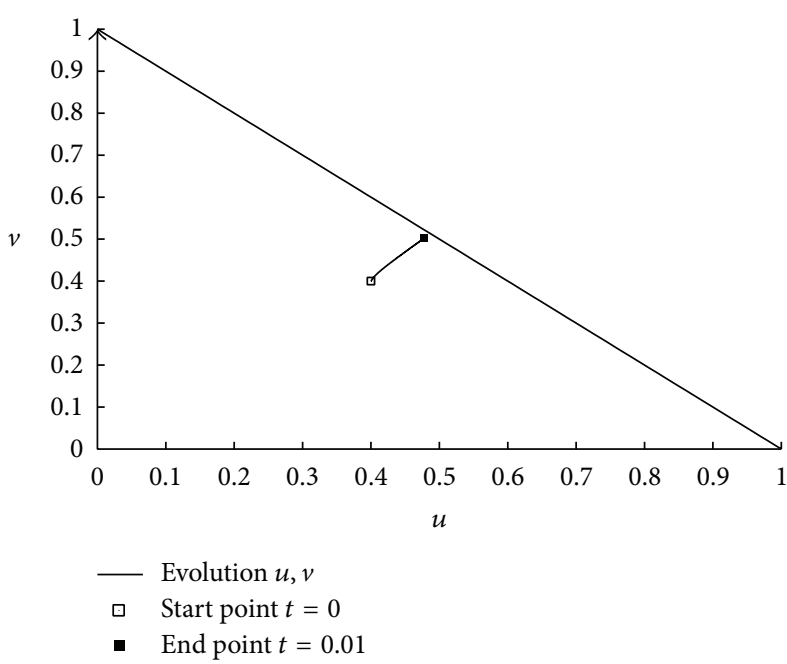

(a)

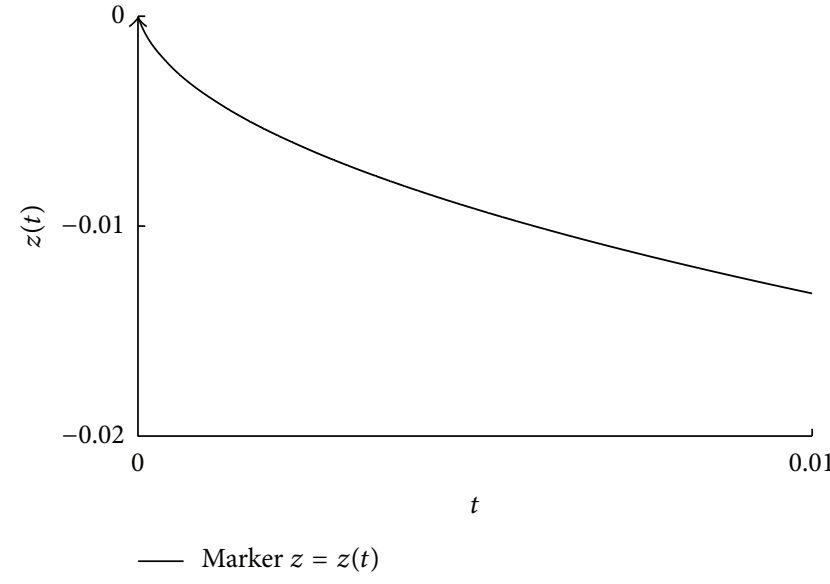

(b)

Figure 2: Evolution of concentrations $u, v$ (a) and experimental position of marker $z=z(t)$ for $0 \leq t \leq 0.01$ (b).

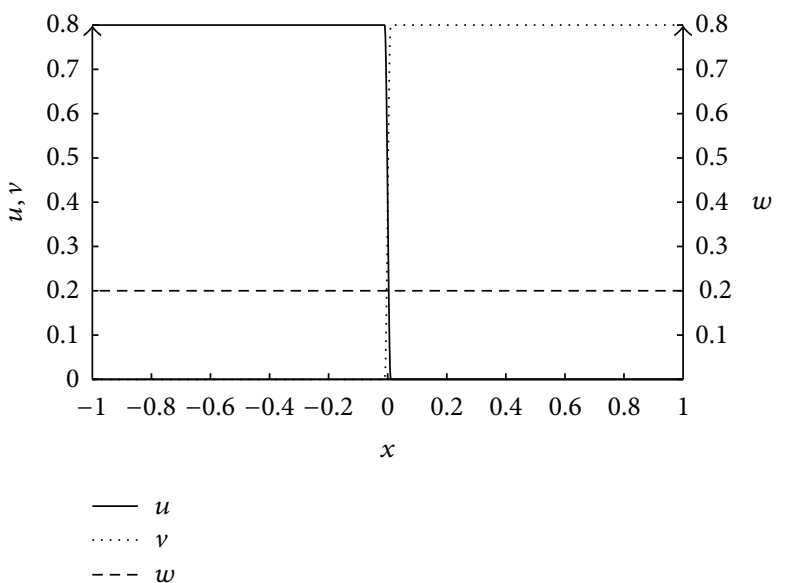

(a)
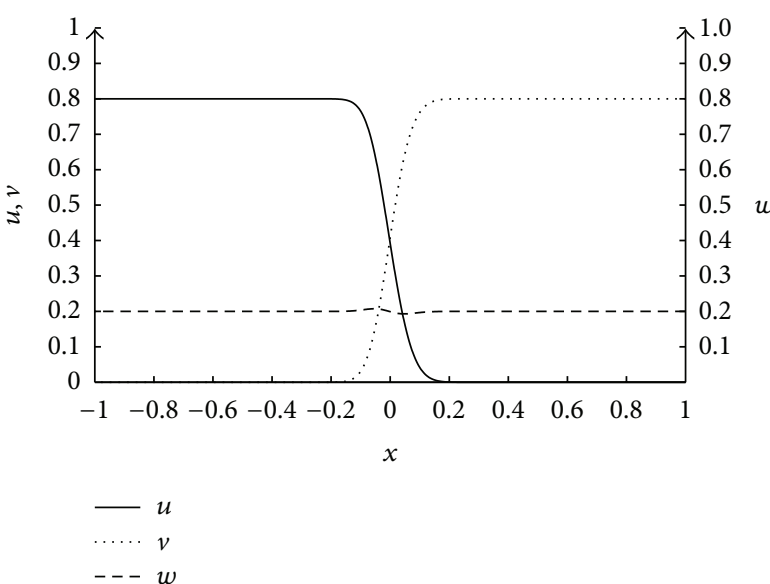

Figure 3: Experimental values of $u, v$, and $w$ for $t=0.01, h=.002$.

marker denoted by $z$ (Figure 2(b)) forms a trajectory in $(u, v)$ plane shown in Figure 2(a). This object traces the evolution of concentrations of diffunding substances $u, v$.

Numerical Example 2. Set $L=1, D_{1}=0.2, D_{2}=0.15, D_{3}=$ 0.08 and the initial distribution of $w$ is constant:

$$
\begin{gathered}
u(0, x)=\Psi(100 x)+\frac{5}{11} \mathbf{1}_{B}(x)+\frac{10}{11} \mathbf{1}_{(.01,1]}(x), \\
v(0, x)=-\Psi(100 x)+\frac{5}{11} \mathbf{1}_{B}(x)+\frac{10}{11} \mathbf{1}_{[-1,-.01)}(x), \\
w(0, x)=\frac{1}{11}, \\
\Psi(x)=\left(\frac{30}{176} x^{5}-\frac{50}{88} x^{3}+\frac{150}{176} x\right) \mathbf{1}_{[-1,1]}(x),
\end{gathered}
$$

for $x \in[-1,1]$ where $\mathbf{1}_{B}$ is the characteristic function of $B:=$ $[-.01, .01]$. Note that $\left(u_{0}, v_{0}, w_{0}\right) \in \mathfrak{X}_{0}$ with $M=M_{0}=30$. In Figure 3(a) we give initial values of the solution $(u, v, w)$ of (8) with the initial condition (46) for $h_{t}=2 \times 10^{-6}$ and $h=.002$. Starting with a constant distribution $w_{0}=1 / 11$ (Figure 3(a)) we give experimental values of $u, v, w$ for $t=$ 0.01 and $h=.002$ (Figure 3(b)). It is seen that diffusion causes a perturbation for $w$. This observation is coherent with engineers' practice.

Our numerical experiments confirm the theory and stability analysis. It is seen that the proposed numerical method is stable, mass-conservative, and dissipative. Its solutions do not leave the interval $[0,1]$. Since our stability analysis relies on the $M$-Lipschitz condition for $u, v, w$, we show (numerically) that the Lipschitz constants for $u, v, w$ decrease in time. We focus on numerical Example 1. Denote these timedependent constants by $M_{u}(t), M_{v}(t), M_{w}(t)$, respectively, and 

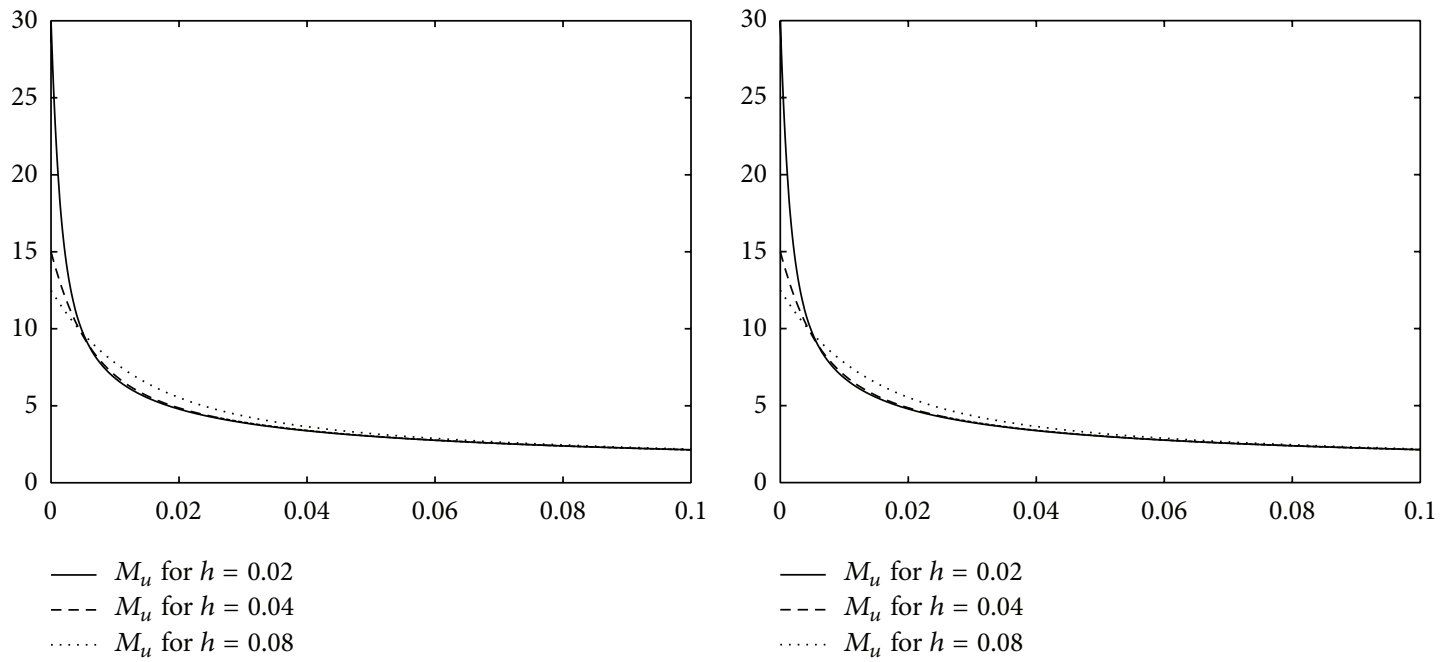

(a)

(b)

FIGURE 4: Experimental values of Lipschitz constants $M_{u}(t)$ and $M_{v}(t)$ for $h=.002, h=.004$, and $h=.008$.
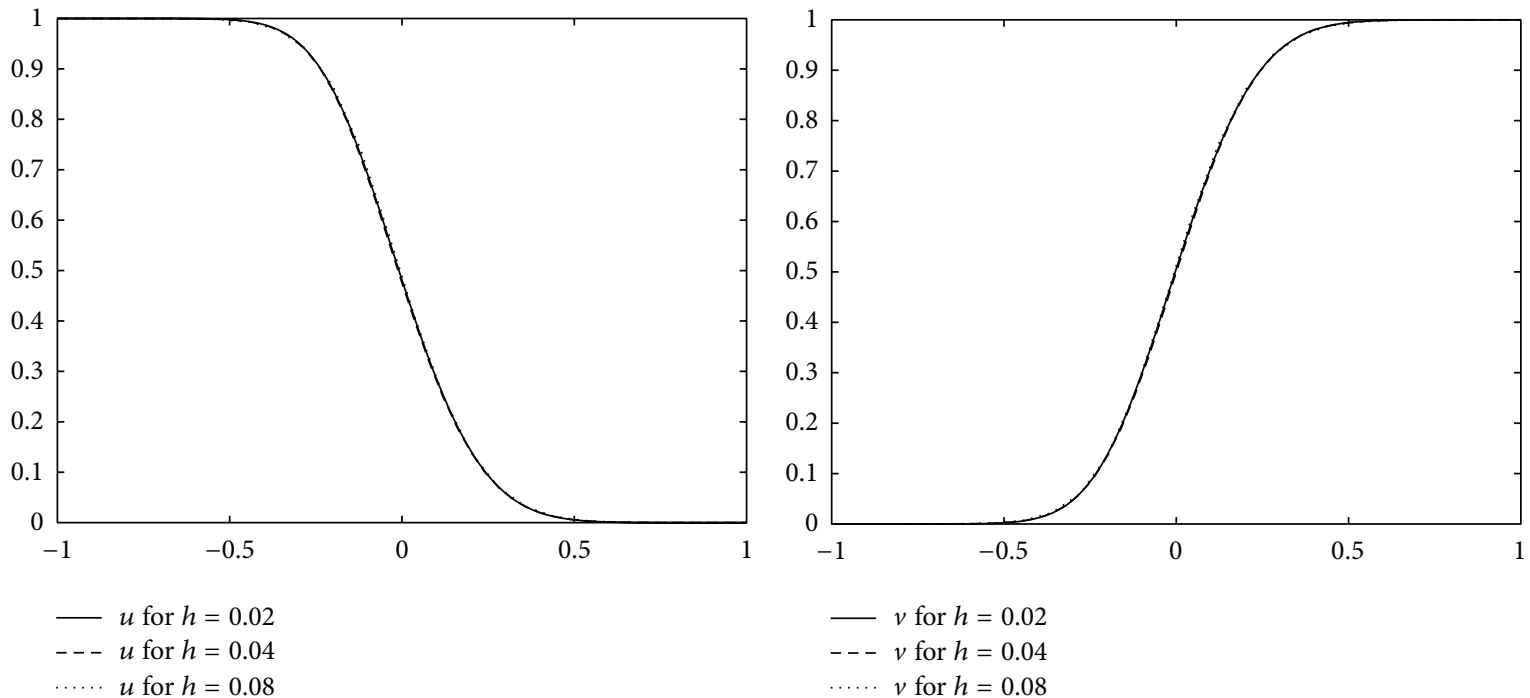

(a)

(b)

FIGURE 5: Experimental values of $u$ and $v$ for $h=.002, h=.004$, and $h=.008$.

show them in Figure 4: $M_{u}$ (a) and $M_{v}$ (b). Considering the same numerical example we demonstrate convergence in Figure 5 where numerical solutions for different steps $h=$ $.008, h=.004$, and $h=.002$ are very close to each other. We take the concentrations $u$ (a) and $v(\mathrm{~b})$.

\section{Appendix}

We formulate a Gronwall type lemma, which was applied in Section 4 .
Lemma A.1. Suppose that $C \geq 0, K \geq 0$

$$
z(t) \leq \int_{0}^{t} \frac{C}{\sqrt{t-s}}[z(s)+p(s)] d s
$$

and $0 \leq p(s) \leq K s^{m}$. Then there is a positive constant $\widetilde{K}$ such that

$$
z(t) \leq \widetilde{K} t^{m+1 / 2}
$$

for $t \in[0, T], 1-2 C T^{1 / 2}>0$. 
Since the right-hand sides of system (8) are polynomials, we can apply the Cauchy-Kovalevskaya theorem. Suppose that solutions of (8) are given by

$$
u^{(j)}(t)=\sum_{k=0}^{\infty} t^{k} u_{k}^{(j)}, \quad v^{(j)}(t)=\sum_{k=0}^{\infty} t^{k} v_{k}^{(j)}
$$

If we differentiate these expressions with respect to $t$ we get the obvious recursive relations for $u_{k+1}^{(j)}$ and $v_{k+1}^{(j)}$. One can prove by induction on $k$ that

$$
\begin{array}{r}
\left|u_{k}^{(j)}\right| \leq C_{*} Q^{k}, \quad\left|v_{k}^{(j)}\right| \leq C_{*} Q^{k} \\
\text { for } k \geq 0, \quad j=-N, \ldots, N,
\end{array}
$$

where

$$
\frac{4 \max \left\{D_{1}, D_{2}\right\}}{(k+1) h^{2}}+\frac{8\left(D_{1}^{\prime}+D_{2}^{\prime}\right) C_{*}}{h^{2}} \leq Q .
$$

This means that the radius of convergence of the above series is lower bounded by $h^{2} / C_{2}$, where

$$
C_{2}=\frac{1}{4 \max \left\{D_{1}, D_{2}\right\}+8\left(D_{1}^{\prime}+D_{2}^{\prime}\right) C_{*}} .
$$

\section{Conflict of Interests}

The authors declare that there is no conflict of interests regarding the publication of this paper.

\section{Acknowledgment}

This work was supported by the National Science Center (Poland) decision no. DEC-2011/02/A/ST8/00280.

\section{References}

[1] A. D. Smigelskas and E. O. Kirkendall, "Zinc diffusion in alpha brass," Transactions of the AIME, vol. 171, pp. 130-142, 1947.

[2] L. S. Darken, "Diffusion, mobility and their interrelation through free energy in binary metallic systems," Transactions of the AIME, vol. 175, pp. 184-201, 1948.

[3] M. Danielewski and B. Wierzba, "Thermodynamically consistent bi-velocity mass transport phenomenology," Acta Materialia, vol. 58, pp. 6717-6727, 2010.

[4] H. Brenner, "Fluid mechanics revisited," Physica A: Statistical Mechanics and its Applications, vol. 370, no. 2, pp. 190-224, 2006.

[5] M. J. H. van Dal, M. C. L. P. Pleumeekers, A. A. Kodentsov, and F. J. J. van Loo, "Intrinsic diffusion and Kirkendall effect in $\mathrm{Ni}-$ Pd and Fe-Pd solid solutions," Acta Materialia, vol. 48, no. 2, pp. 385-396, 2000.

[6] F. J. J. van Loo, B. Pieraggi, and R. A. Rapp, "Interface migration and the kirkendall effect in diffusion-driven phase transformations," Acta Metallurgica et Materialia, vol. 38, no. 9, pp. 1769-1779, 1990.

[7] A. Paul, M. J. H. van Dal, A. A. Kodentsov, and F. J. J. Van Loo, "The Kirkendall effect in multiphase diffusion," Acta Materialia, vol. 52, no. 3, pp. 623-630, 2004.
[8] M. J. H. van Dal, A. M. Gusak, C. Cserháti, A. A. Kodentsov, and F. J. J. van Loo, "Microstructural stability of the Kirkendall plane in solid-state diffusion," Physical Review Letters, vol. 86, no. 15, pp. 3352-3355, 2001.

[9] M. J. H. van Dal, A. M. Gusaki, C. Cserháti, A. A. Kodentsov, and F. J. J. van Loo, "Spatio-temporal instabilities of the Kirkendall marker planes during interdiffusion in $\beta^{\prime}$-AuZn," Philosophical Magazine A: Physics of Condensed Matter, Structure, Defects and Mechanical Properties, vol. 82, no. 5, pp. 943-954, 2002.

[10] W. J. Boettinger, J. E. Guyer, C. E. Campbell, and G. B. McFadden, "Computation of the Kirkendall velocity and displacement fields in a one-dimensional binary diffusion couple with a moving interface," Proceedings of the Royal Society A: Mathematical, Physical and Engineering Sciences, vol. 463, no. 2088, pp. 3347-3373, 2007.

[11] M. Danielewski, K. Holly, and W. Krzyżański, "Interdiusion in rComponent $(r \geq 2)$ one dimensional mixture showing constant concentration," Computer Methods in Materials Science, vol. 8, pp. 31-46, 2008.

[12] K. Holly and M. Danielewski, "Interdiffusion and freeboundary problem for r-component (r2) one-dimensional mixtures showing constant concentration," Physical Review B, vol. 50, no. 18, pp. 13336-13346, 1994.

[13] W. Hundsdorfer and J. Verwer, Numerical Solution of TimeDependent Advection-Diffusion-Reaction Equations, Springer, Berlin, Germany, 2003.

[14] W. E. Schiesser, The Numerical Method of Lines, Academic Press, San Diego, Calif, USA, 1991.

[15] A. Vande Wouwer, Ph. Saucez, and W. E. Schiesser, Adaptative Method of Lines, Chapman \& Hall/CRC, New York, NY, USA, 2001.

[16] A. Al-Rabeh, "Towards a general integration algorithm for time-dependent one-dimensional systems of parabolic partial differential equations using the method of lines," Journal of Computational and Applied Mathematics, vol. 42, no. 2, pp. 187198, 1992.

[17] I. Babuška, M. Feistauer, and P. Šolín, "On one approach to a posteriori error estimates for evolution problems solved by the method of lines," Numerische Mathematik, vol. 89, no. 2, pp. 225-256, 2001.

[18] M. Danielewski, R. Filipek, K. Holly, and B. Bozek, "Interdiffusion in multicomponent solid solutions, the mathematical model for thin films," Physica Status Solidi A, vol. 145, pp. 339350, 1994.

[19] W. Walter, Differential and Integral Inequalities, Springer, New York, NY, USA, 1970.

[20] O. A. Ladyženskaja, V. A. Solonnikov, and N. N. Uraltseva, Linear and Quasilinear Equations of Parabolic Type, vol. 23 of Translations of Monographs, American Mathematical Society, Providence, RI, USA, 1968. 


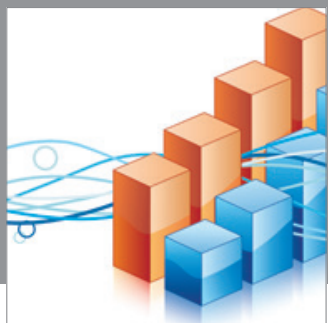

Advances in

Operations Research

mansans

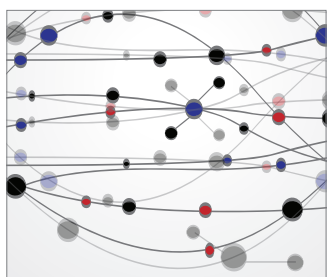

The Scientific World Journal
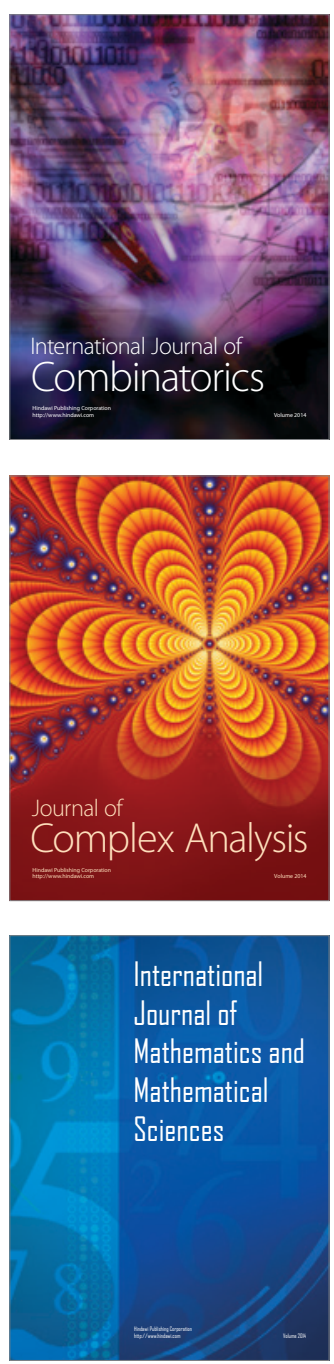
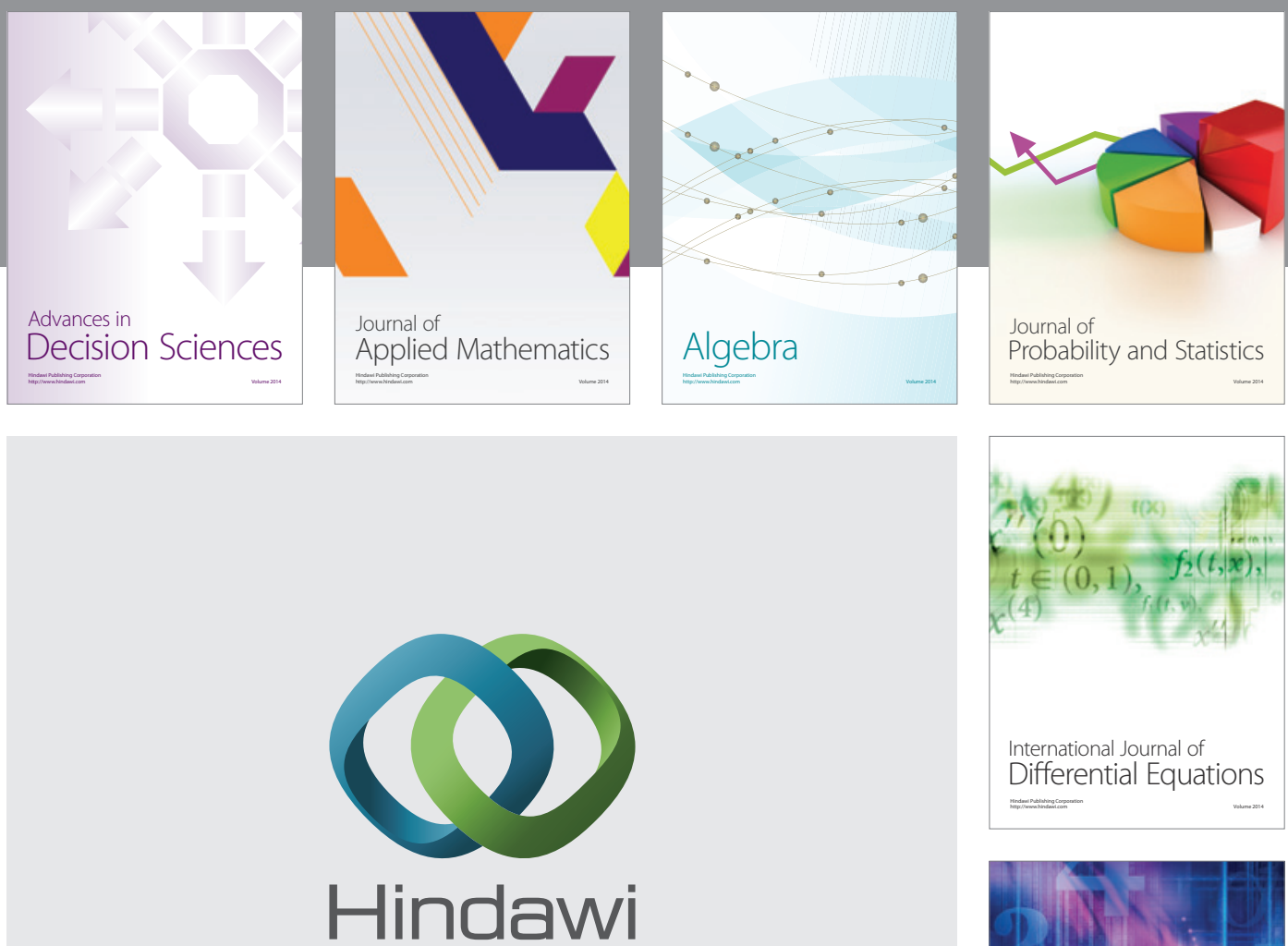

Submit your manuscripts at http://www.hindawi.com
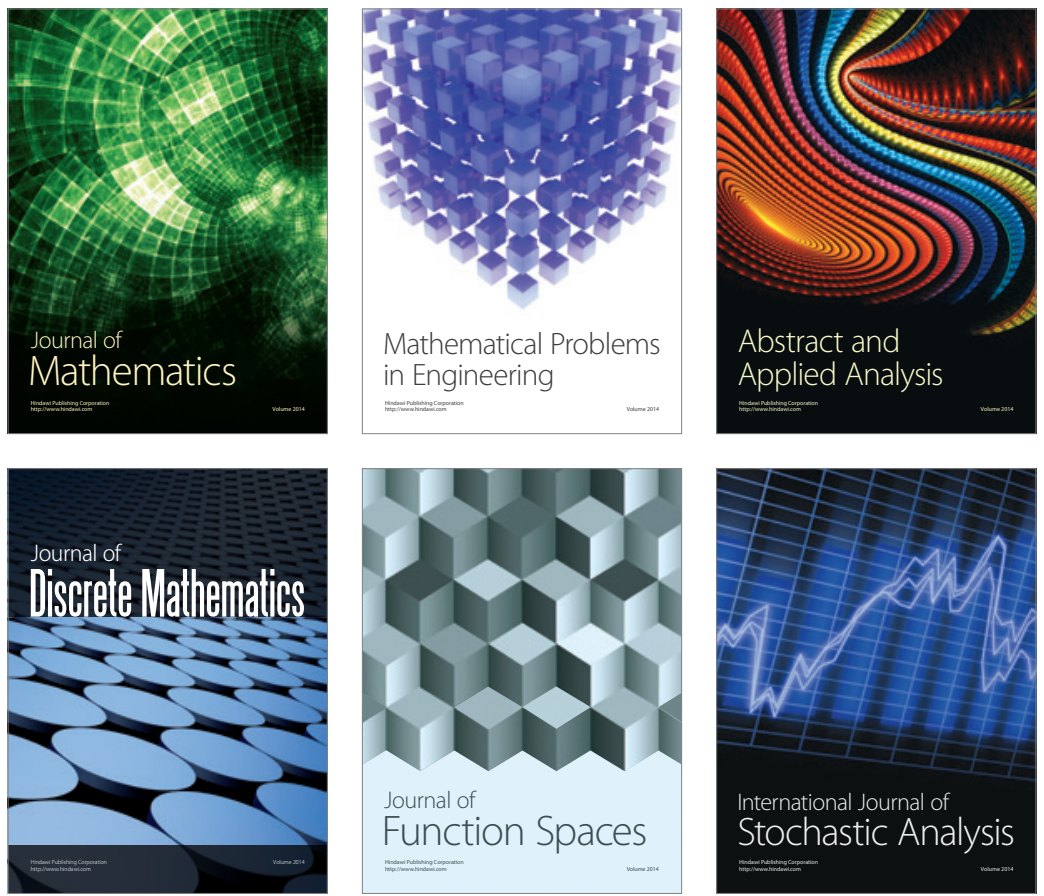

Journal of

Function Spaces

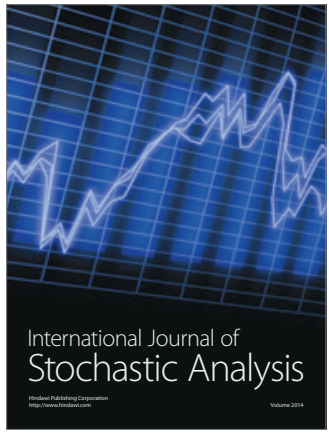

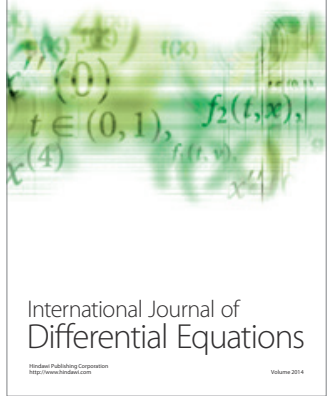
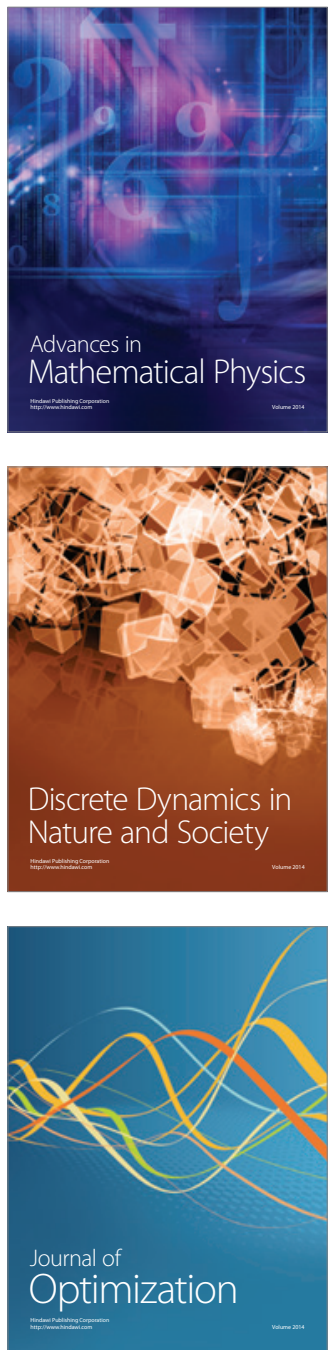